\title{
Occurrences and Narratives as Constraints in the Branching Structure of the Situation Calculus.
}

\author{
Javier A. Pinto* \\ Departamento de Ciencia de la Computación \\ Escuela de Ingeniería \\ Pontificia Universidad Católica de Chile \\ jpinto@ing.puc.cl
}

August 29, 1996

\begin{abstract}
The Situation Calculus is a logic of time and change in which there is a distinguished initial situation $S_{0}$ and all other situations arise from the different sequences of actions that might be performed starting in the initial one. Within this framework, it is difficult to incorporate the notion of an occurrence, since all situations after the initial one are hypothetical. These occurrences are important, for instance, when one wants to represent narratives. There have been proposals to incorporate the notion of an action occurrence in the language of the Situation Calculus, namely Miller and Shanahan's work on narratives [22] and Pinto and Reiter's work on actual lines of situations [27, 29]. Both approaches have in common the idea of incorporating a linear sequence of situations into the tree described by theories written in the Situation Calculus language. Unfortunately, several advantages of the Situation Calculus are lost when reasoning with a narrative line or with an actual line of occurrences.

In this paper we propose a different approach to dealing with action occurrences and narratives, which can be seen as a generalization of narrative lines to narrative trees. In this approach we exploit the fact that, in the discrete Situation Calculus [13], each situation has a unique history. Then, occurrences are interpreted as constraints on valid histories. We argue that this new approach subsumes the linear approaches of Miller and Shanahan's, and Pinto and Reiter's. In this framework, we are able to represent various kinds of occurrences; namely, conditional, preventable and non-preventable occurrences. Other types of occurrences, not discussed in this article, can also be accommodated.
\end{abstract}

\section{Introduction}

The Situation Calculus [18] is one of the standard logical languages used in Artificial Intelligence to write theories of action and change. The original language is based on an ontology in which actions are instantaneous and non-concurrent. This language has been the focus of much interest in the Knowledge Representation community, and has motivated a great deal of research (e.g., $[3,7,2,14,22,24])$. The Situation Calculus can be considered to be a branching time temporal

*This work has been partly funded by the Fondecyt project number 195-0885. 
logic. Indeed, the structure of situations in the Situation Calculus embodies all possible courses of action that can be taken starting from an initial situation. This view leads to considering every situation as being hypothetical, in the sense that the situation would arise only if the actions that lead to it were actually executed. An important advantage of this approach, over linear time approaches, is that one can reason about these different possible alternatives, and this reasoning can be done within the language. Indeed, in the Situation Calculus we have, at the object level, situations that arise from performing a sequence of actions $a_{1}, a_{2}, \ldots, a_{n}$, along with other situations that arise from performing totally different sequences of actions $a_{1}^{\prime}, a_{2}^{\prime}, \ldots, a_{m}^{\prime}$ (all starting, possibly, in the same initial situation). Having the resulting situations available, at the object level, is not possible in linear time logics. In fact, the occurrence of a completely specified sequence of actions precludes reasoning about other possible sequences of actions.

One advantage of linear time approaches, such as the Calculus of Events [8], Allen's Temporal Logic [1] and other logics of time is that it is easy to state knowledge regarding action occurrences. Indeed, this logics are only concerned with the way in which the world actually unfolds. Therefore, if one knows that some action occurs, it is possible to say that this action is related to a time point through some occurrence predicate. This allows one to specify the state of the world at any given time. In contrast, in branching time logics, this is not always clear what one means by an action occurrence. In particular, in the Situation Calculus approach, it is difficult to represent the notion of an occurrence, which leads to difficulties in representing:

Narratives: A possibly incomplete specification of actions or events that are known to occur at specific time points.

Triggers: The specification of actions or events that are triggered by the occurrence of other actions or events.

The goal of this work is to add to the Situation Calculus the expressiveness required to represent occurrences and narratives and be able to reason hypothetically with them.

To our knowledge, there have been two approaches to incorporate narratives in the Situation Calculus. In [22], Miller and Shanahan introduce a time line and narratives are described in terms of this time line. Additionally, they associate one situation with each action in the narrative. Also, they introduce a mapping Sit from time points to situations, such that each time point (in the positive real time line) is associated with a unique situation. A different, but related approach, is taken by Pinto and Reiter [29]. In this second approach, one path in the tree, which starts at $S_{0}$, is selected. This path is called the actual path of situations and is meant to identify a path in the tree that represents what really happens in the world. In [26], a circumscription based formalization is used to select preferred interpretations for the actual line. Each situation in this path is assigned a time point. There is a clear correspondence between the two approaches. Indeed, the mapping Sit of Miller and Shanahan's can be equated with the actual situations of Pinto and Reiter's. Furthermore, Shanahan has shown that, under suitable conditions, both approaches yield the same results [37]. In these two approaches, circumscription axioms are used in order to formalize the assumption that no events occur unless they must occur. As Reiter has observed [34], this leads to the premature minimization problem, discussed below.

Unfortunately, approaches based on the notion of this actual or narrative line have certain undesirable properties. For instance, let us assume that an agent's behavior is specified using the Situation Calculus with an actual line of situations. Thus, the specification describes the way in which the world actually evolves. Unfortunately, as in linear time theories, it is not possible to hypothesize about the different courses of action that the agent might have taken, and that are not actual. Such hypothetical reasoning has to be performed at a meta-level. 
In our current approach to modeling occurrences within the Situation Calculus we make use of a legal predicate for situations. This predicate was introduced by Reiter in order to constrain the valid situations. The set of legal situations forms a tree rooted at $S_{0}$. Legal situations are constrained in several ways. For instance, the precondition axioms for actions enforce the constraint that an action whose preconditions are not satisfied does not lead to a legal situation. Also, Reiter introduced an approach to handling natural actions [30] that provides further constraints for legal situations. In this paper, we propose to view occurrence statements as constraints on the legal situations in the tree. These constraints can be seen as "pruning" statements that tell us what situations in the tree are suitable hypothetical futures. For instance, a narrative will become a set of sentences specifying which paths along the tree of situations can be considered valid developments.

Our approach to handle occurrences in the Situation Calculus has several advantages. For instance, it allows for the representation of simple non-preventable occurrences and narratives. Also, it allows for the representation of triggers [38] and preventable occurrences. Furthermore, the advantages of the original Situation Calculus are not lost. In particular, we are still able to do deductive planning in the presence of occurrences. Moreover, this approach does not suffer from what Reiter calls premature minimization problem [34]:

"[This problem] amounts to the assumption that all action occurrences are either specified as part of the axiomatization, or are inferable from it. Closure, in the form of minimization of action occurrences, is enforced by suitably circumscribing these axioms."

In fact, we show that our axiomatization does not preclude one from considering action occurrences that are not entailed by the theory. However, we can do even better. Indeed, we show that it is possible, within the first order logical language, to characterize sequences of actions that are preferred using some minimality assumptions. Interestingly, we are able to define this notion of preferred development within the language of the Situation Calculus without having to appeal to circumscription axioms.

The reader might wonder why we chose the Situation Calculus as a formal framework to build theories for the representation of knowledge about action and change. To date, there is no broadly accepted formalism for the representation of knowledge of this nature. However, the Situation Calculus has emerged as one candidate approach. There is a concerted effort to enrich the language with the ability to represent a variety of types of knowledge relevant for dynamic domains. For instance, there are proposals to deal with concurrency [14, 24, 23, 34], proposals to extend the Situation Calculus to deal with continuous change [24, 25, 20, 34], proposals to add epistemic features [35, 10], etc. Furthermore, the Situation Calculus has been proposed as the basis for the development of the agent programming language $G O L O G[9,11]$. The work reported in this article forms part of this research tradition, started by John McCarthy in [16].

The structure of this paper is as follows: In section 2, we present the theoretical framework on which the rest of the paper is based. In section 3, we present our approach to modeling occurrences and triggers. In section 4, we present applications of this approach to knowledge representation problems. In section 5, we compare our approach to approaches based on a time line. Finally, in section 6 , we present our concluding remarks and discuss future developments of these ideas. 


\section{Theoretical Framework}

In this chapter we present the formal framework that will be used in the rest of the paper. The basic language and axiomatization are extensions to the Situation Calculus formalism proposed in $[24,30,34]$. The main novelty is that we introduce new predicates in the language that allow us to refer to finite paths or histories in the Situation Calculus trees. In the following section, these new predicates will be used to define different notions of legality of situations in the presence of occurrence sentences.

\subsection{Language}

The Situation Calculus we use is a sorted first order language extended with a second order induction axiom to characterize the space of situations. We distinguish the sorts $\mathcal{A}, \mathcal{C}, \mathcal{S}$ and $\mathcal{T}$ for primitive actions, concurrent actions, situations and time. We also use a sort $\mathcal{D}$ for other objects. The sort $\mathcal{T}$ ranges over the positive real numbers. We use the convention that all variable symbols start with lower case letters, whereas constant symbols start with upper case letters. Also, we use the Greek letter $\varphi$ as a second order predicate variable, which is only used in the specification of the induction axiom for situations given in section 2.3. The sort of the variables and constants should be inferable from the context. In formulas, we assume that all free variables are universally quantified from the outside. The sort $\mathcal{C}$ corresponds to sets of primitive actions (following [34]). We take the standard interpretation for sets and don't axiomatize them.

\section{Function and Predicate Symbols}

start $: \mathcal{S} \rightarrow \mathcal{T}$. If $s$ is a situation term, then $\operatorname{start}(s)$ denotes the time at which that situation starts.

do: $\mathcal{C} \times \mathcal{S} \rightarrow \mathcal{S}$. If $c$ denotes a concurrent action and $s$ denotes a situation, then $d o(c, s)$ denotes the situation that results from performing $c$ in $s$. For notational convenience, the term $d o\left(c_{n}, d o\left(\ldots, d o\left(c_{1}, S_{0}\right) \ldots\right)\right)$ will be written as $d o\left(\left[c_{1}, \ldots, c_{n}\right], S_{0}\right)$.

$\in \subseteq \mathcal{A} \times \mathcal{C}$. This is the standard membership operator for sets.

hasOccurred $\subseteq(\mathcal{A} \cup \mathcal{C}) \times \mathcal{T} \times \mathcal{S}$. The first argument of hasOccurred can be either a primitive action or a concurrent action. hasOccurred $(x, t, s)$ would be true if action $x$ (primitive or concurrent) occurred at time $t$ on the path that leads from $S_{0}$ to $s$.

$\prec \subseteq \mathcal{S} \times \mathcal{S}$. If $s_{1}$ and $s_{2}$ denote situations, then $s_{1} \prec s_{2}$ is true if some sequence of concurrent actions leads from $s_{1}$ to $s_{2}$. We use the abbreviation:

$$
s_{1} \preceq s_{2} \equiv s_{1} \prec s_{2} \vee s_{1}=s_{2} .
$$

Poss $\subseteq(\mathcal{A} \cup \mathcal{C}) \times \mathcal{S}$. The first argument of Poss can be either a primitive action or a concurrent action. If $x$ is an action (primitive or concurrent) and $s$ a situation, then $\operatorname{Poss}(x, s)$ is true if the action $x$ is possible in $s$ (i.e., if the action preconditions are satisfied in the situation denoted by $s$ ).

$<\subseteq \mathcal{S} \times \mathcal{S}$. If $s_{1}$ and $s_{2}$ denote situations, then $s_{1}<s_{2}$ is true if some sequence of possible concurrent actions leads from $s_{1}$ to $s_{2}$. We use the abbreviation:

$$
s_{1} \leq s_{2}=s_{1}<s_{2} \vee s_{1}=s_{2} .
$$


$<\subseteq \mathcal{T} \times \mathcal{T}$. We overload this symbol and use it as the standard comparison operator for reals (the abbreviation $\leq$ is also used).

legal $_{\text {poss }}$, legal $\subseteq \mathcal{S}$. These predicate are used to characterize those situations that are valid; a situation is considered valid if and only if it obeys all the preconditions and occurrence constraints.

proper $\subseteq \mathcal{S}$. Is an auxiliary predicate used to define legal situations.

Sit $\subseteq \mathcal{T} \times \mathcal{S} \times \mathcal{S}$. The literal $\operatorname{Sit}\left(t, s, s_{h}\right)$ is intended to mean that $t$ falls in the situation $s$ in the path that leads from $S_{0}$ to $s_{h}$. The predicate Sit we use here is not related to the the mapping Sit used in [22].

occurrence predicates. In section 3, we introduce several notions of occurrence. In each case, a different occurrence predicate is introduced.

\subsection{Representational Restrictions}

In this paper, we consider a Situation Calculus in which actions are considered to be instantaneous. As discussed elsewhere (e.g., [23]), this restriction does not limit the representational power of the language, since actions with durations can be model-led as a pair of instantaneous actions, one which starts the original action, and one that finishes it. Furthermore, one can easily introduce a new sort of actions with durations with mappings to instantaneous actions that start and finish them.

Also, we ignore precondition interactions and concurrent effects or cancellations. Precondition interactions arise when the concurrent execution of two actions is precluded in spite of the fact that each action can be executed independently. The concurrent effects arise when the concurrent execution of two actions brings about effects that neither action would have when executed individually. Finally, concurrent cancellation arises when the effect of one action is canceled when the action is concurrently executed with another. These problems are discussed in $[23,24]$.

The issues discussed in this paper are orthogonal to those mentioned above. Therefore, any approach to deal with precondition interactions and concurrent effects/cancellation should integrate well with the theory presented in this paper.

\subsection{Basic Situation Calculus Axiomatization}

In the Situation Calculus there is an initial situation, denoted by the special constant symbol $S_{0}$. Given an arbitrary situation $s$, a new situation is obtained by performing some concurrent action $c$ in $s$. Following Reiter [31], we use an induction axiom in order to force the set of situations to form a tree rooted at $S_{0}$. Thus, an arbitrary situation $s$ identifies a unique sequence of concurrent actions that leads from $S_{0}$ to $s$. Each situation $s$ in the tree has a unique starting time [29]; this time corresponds to the time of the last action in the sequence leading to $s$. On the other hand, there is no unique ending time for a situation. In fact, in [29], Pinto and Reiter define an end predicate that takes an action and a situation. Roughly, the end of a situation $s$ with respect to an action $c$ corresponds to the start time of the situation $d o(c, s)$. These multiple ending times arise from the fact that, in the Situation Calculus, the future is branching. 
The basic axioms for situations, explained below, are:

$$
\begin{aligned}
& (\forall \varphi) \cdot\left[\varphi\left(S_{0}\right) \wedge(\forall s, c)(\varphi(s) \supset \varphi(d o(c, s)))\right] \supset(\forall s) \varphi(s), \\
& \operatorname{do}\left(c_{1}, s_{1}\right)=\operatorname{do}\left(c_{2}, s_{2}\right) \supset c_{1}=c_{2}, \\
& s_{1} \prec d o\left(c, s_{2}\right) \equiv s_{1} \preceq s_{2}, \\
& s_{1} \prec s_{2} \supset \neg s_{2} \prec s_{1}, \\
& \operatorname{Poss}(c, s) \equiv(\forall a)[a \in c \supset \operatorname{Poss}(a, s)], \\
& \operatorname{Poss}(c, s) \supset \operatorname{start}(s)<\operatorname{start}(\operatorname{do}(c, s)) .
\end{aligned}
$$

The first axiom is a second order induction axiom, similar to Peano's induction axiom used to characterize the natural numbers. This axiom states that the only situations that exist are $S_{0}$, the initial situation, and all the situations that are reachable from $S_{0}$ by performing sequences of actions starting in $S_{0}$. It is important to point out that this is the only use we make of second order logic in this article. Furthermore, in the same manner as is done with the natural numbers, we could replace this axiom with an axiom schema and work with a standard interpretation. In theorem proving, the axiom can be left implicit in the proof strategy, as done with $R R L$ (Rewrite Rule Laboratory [6]) in [2], where induction is used in order to mechanically prove integrity constraints from a Situation Calculus specification of database transactions.

Axiom (2) ensures that the structure of situations forms a tree, and roughly corresponds to Shanahan's arboreality axiom [37]. Axioms (3) and (4) define the relation $\preceq$. The literal $S_{1} \prec S_{2}$ is true if there is a path from $S_{1}$ to $S_{2}$ in the Situation Calculus tree; i.e., $S_{2}$ is reached by performing a sequence of actions starting from $S_{1}$.

Axiom (5) states that a concurrent action is possible iff all its constituent actions are individually possible. We assume that the predicate Poss for simple actions is completely specified by domain axioms.

As mentioned before, the function start denotes the starting time of a situation. Axiom (6) ensures that the situations along a path are chronologically ordered. This ordering is only enforced for situations that are obtained when performing actions that are possible.

\subsection{Situation Legality}

In the previous sub-section, we presented the basic axiomatization that defines the universe of situations $\mathcal{S}$ in terms of the initial situation $S_{0}$ and the set of concurrent actions $\mathcal{C}$. Notice that this universe of situations is defined without taking into consideration whether or not the situations were actually possible. That is, there are situations that are reached by performing sequences of actions that include sub-actions that are not possible. Our objective is to specify which situations in the Situation Calculus tree can be regarded as viable or legal given knowledge regarding action preconditions, laws of physics, occurrence statements, etc. In [33], Reiter defines a first notion of legality which basically says that a situation $s$ is legal if it can be reached by performing actions in $S_{0}$ and all the actions leading to $s$ are performed in situations in which their preconditions are met. This motivates the following:

Definition 2.1 A situation s is considered legal with respect to the action preconditions, denoted legal ${ }_{\text {poss }}$, if all the actions that lead from $S_{0}$ to $s$ have been done in situations in which they are possible. Thus, legal $l_{\text {poss }}$ is defined as:

$$
\operatorname{legal}_{\text {poss }}(s) \equiv s=S_{0} \vee\left(\forall c, s^{\prime}\right) d o\left(c, s^{\prime}\right) \preceq s \supset \operatorname{Poss}\left(c, s^{\prime}\right)
$$


Reiter defines legality with the predicate $<$, which he axiomatizes as follows:

$$
\begin{gathered}
\neg s<S_{0}, \\
s<d o\left(c, s^{\prime}\right) \equiv \operatorname{Poss}\left(c, s^{\prime}\right) \wedge s \leq s^{\prime} .
\end{gathered}
$$

The relationship between legal poss $_{\text {and }}<$ is straightforward. In fact, we have the following simple observation:

Observation 2.1 From axioms (1)-(9) it follows that:

$$
\text { legal }_{\text {poss }}(s) \equiv S_{0} \leq s .
$$

PROOF: The proof is a direct application of the induction axiom (1) with

$$
\varphi(s)=\left(\text { legal }_{\text {poss }}(s) \equiv S_{0} \leq s\right) .
$$

The legality predicates - legal poss $_{\text {, }}$ along with other legal predicates - are introduced as a tree pruning mechanism. Thus, legal poss $_{\text {is }}$ a first step towards specifying which part of the Situation Calculus tree corresponds to valid developments. As a next step in this pruning process, we can incorporate the notion of natural actions [24, 30, 34]. Natural actions are the result of the laws of physics. For instance, the action hit Floor $(\mathrm{Ball})$ might be regarded as natural if $\mathrm{Ball}$ is falling in such a way that it will hit the floor at a certain time. See [34] for an approach to introducing natural actions as constraints on the legal situations. In this article we ignore natural actions. However, it should not be difficult to incorporate them in our framework as further constraints on the legality of situations.

\subsection{Situations as Histories or Paths}

The notion of a history, within the Situation Calculus, corresponds to the sequence of actions that leads to a situation starting from $S_{0}$. Also, we refer to this as a partial path within the Situation Calculus tree. A path in the Situation Calculus tree corresponds to an infinite sequence of situations that start in $S_{0}$, a partial path is a prefix of infinitely many paths. Thus, the notion of path corresponds to McDermott's notion of chronicle[19]. Any situation $s$ identifies a unique partial path that starts at $S_{0}$ and ends with $s$. In fact, any situation in the domain $\mathcal{S}$ is fully identified with a sequence of actions. For instance, the sequence $c_{1}, c_{2}, \ldots, c_{n}$ uniquely identifies the situation $S_{n}$, where:

$$
S_{n}=d o\left(\left[c_{1}, \ldots, c_{n}\right], S_{0}\right)
$$

Thus, all the actions $c_{1}, \ldots, c_{n}$ can be said to belong to or have occurred before $S_{n}$, and they correspond to the history of situation $d o\left(\left[c_{1}, \ldots, c_{n}\right], S_{0}\right)$. It turns out that one essential feature of the Situation Calculus, not available in linear time languages, is that there are terms denoting situations, which also denote histories. Thus, a situation $s$ corresponds to a single history or linear time description of one possible evolution of the world starting from $S_{0}$. A situation $s^{\prime}$ is said to appear or belong to a history $s$ iff $s^{\prime} \prec s$. Thus, $S_{0}$ belongs to all histories (except to the empty history, which corresponds to $S_{0}$ ). Notice that the notion of history comes for free in the Situation Calculus and no added machinery needs to be introduced in the language. 
The reader might be wondering why we introduce new distinctions (history, partial path) in order to refer to the concept of situation. The reason is that we want to emphasize the fact that situations are looked at in a non-traditional manner. It is very common to consider situations as snapshots that don't have an inherent history. We use the term history in order to emphasize that we are looking at an aspect of a situation which is normally not taken into account.

The branching nature of time in the Situation Calculus makes it difficult to impose a unique ending time on situations (they do have a unique start time, since time in the Situation Calculus is left linear). On the other hand, in a given history, any situation has a unique starting and a unique ending time. If $s$ and $d o(c, s)$ belong to some history $s^{\prime}$, then $\operatorname{start}(s)$ and $\operatorname{start}(d o(c, s))$ correspond to the starting and ending times of $s$ along the history $s^{\prime}$.

In this paper, we exploit the notion of histories to define occurrences. We do so by defining the notion of a concurrent action $c$ occurring within a history, partial path or situation:

Definition 2.2 We say that a concurrent action c has occurred at time $t$ along the history of $s$ with the predicate hasOccurred as follows:

$$
\text { hasOccurred }(c, t, s) \equiv\left(\exists s^{\prime}\right) \operatorname{do}\left(c, s^{\prime}\right) \preceq s \wedge \operatorname{start}\left(\operatorname{do}\left(c, s^{\prime}\right)\right)=t .
$$

This is extended for primitive actions as:

$$
\text { hasOccurred }(a, t, s) \equiv(\exists c) a \in c \wedge \text { hasOccurred }(c, t, s) .
$$

An interesting property of these definitions is the following:

Observation 2.2 From axioms (1)-(11) it follows that some action c has occurred in a history s if it is equal to the last action in the history, or if it has also occurred in the situation immediately preceding $s$ :

$$
\begin{aligned}
\text { hasOccurred }\left(c, t, \operatorname{do}\left(c^{\prime}, s\right)\right) \equiv & c=c^{\prime} \wedge \operatorname{start}\left(\operatorname{do}\left(c^{\prime}, s\right)\right)=t \vee \\
& \text { hasOccurred }(c, t, s), \\
\operatorname{hasOccurred}\left(a, t, \operatorname{do}\left(c^{\prime}, s\right)\right) \equiv & a \in c^{\prime} \wedge \operatorname{start}\left(\operatorname{do}\left(c^{\prime}, s\right)\right)=t \vee \\
& \text { hasOccurred }(a, t, s) .
\end{aligned}
$$

PROOF: Apply definition (10) and axiom (3).

Also, we have:

Observation 2.3 From axioms (1)-(11) it follows that if two concurrent actions $c$ and $c^{\prime}$ have occurred at the same time in a given history, then both actions are the same:

$$
\text { hasOccurred }(c, t, s) \wedge \text { hasOcurred }\left(c^{\prime}, t, s\right) \supset c=c^{\prime} .
$$

PROOF: From the basic structural axioms (1)-(4) it is possible to show that the structure of situations is left linear, that is:

$$
\left[s_{1} \preceq s \wedge s_{2} \preceq s\right] \supset\left[s_{1} \preceq s_{2} \vee s_{2} \preceq s_{1}\right]
$$

This property, along with (6) and definition (10) lead to the proof. 
Finally, we introduce the following:

Definition 2.3 We say that $\operatorname{Sit}\left(t, s, s_{h}\right)$ is true whenever $s$ corresponds to the situation in the history $s_{h}$ which includes time $t$ (thus $t$ is between the start of $s$ and the end of $s$ along the history $s_{h}$ ). Formally,

$$
\operatorname{Sit}\left(t, s, s_{h}\right) \equiv(\exists c) \operatorname{do}(c, s) \preceq s_{h} \wedge \operatorname{start}(s)<t \wedge t \leq \operatorname{start}(\operatorname{do}(c, s)) .
$$

Using these definitions we have:

Observation 2.4 From axioms (1) to (13) it follows that the situation that includes time t along the history do $(c, s)$, for some situation $s$ and action $c$, is s. I.e.:

$$
\operatorname{Sit}(\operatorname{start}(d o(c, s)), s, \operatorname{do}(c, s)) \text {. }
$$

Furthermore, if two situations include a single time point along some history, then both situations must be the same, i.e.:

$$
\operatorname{Sit}\left(t, s_{1}, s_{h}\right) \wedge \operatorname{Sit}\left(t, s_{2}, s_{h}\right) \supset s_{1}=s_{2} .
$$

PROOF: The first part is straightforward. The second part involves the use of the left linear property of the structure of situations, mentioned in the proof of observation 2.3, and the use of induction along with axiom (6).

\section{Formalizing Action Occurrences}

\subsection{Further Constraints on Legal Situations}

The main purpose of this paper is to deal with the problem of representing the notion of action occurrence in the framework of the Situation Calculus. A solution to the problem of representing action occurrences leads to a solution to the problem of representing narratives and triggers.

In this section, we introduce two new predicates proper $\subseteq \mathcal{S}$ and legal $\subseteq \mathcal{S}$. A situation is said to be a proper history, iff it obeys all the constraints up to the start of the situation (i.e., without looking towards the future of the situation). This predicate is introduced in order to characterize those histories that are legal with respect to the preconditions and that conform to the occurrence specifications (introduced later). proper is a predicate defined in terms of

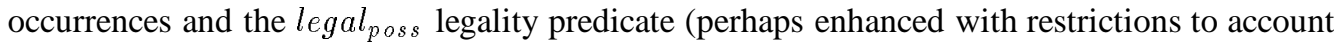
for natural actions). However, for presentation purposes, the specification of proper will be given as a set of implications or necessary conditions for proper. In the end, the set of necessary conditions for proper will be regarded as sufficient conditions as well.

The first necessary condition for a situation to represent a proper partial path is:

$$
\operatorname{proper}(s) \supset \text { legal }_{\text {poss }}(s) .
$$

Thus, actions that are not possible do not lead to proper histories. 
All other constraints on proper will be introduced in the context of the occurrence predicates that are introduced in later sections.

The predicate legal should be taken as a refinement of the legality notion introduced with the legal poss $_{\text {predicate. In fact, if a situation is legal, then it is also legal }}$ poss. We say that a situation is legal if it obeys all constraints, related to action preconditions and occurrence sentences. The definition of legal is done in terms of the proper predicate:

Definition 3.1 A situation or history $s$ is considered to be legal with respect to the action preconditions and occurrence statements if every time after its start there is a proper future history that contains $s$. This is formalized as:

$$
\operatorname{legal}(s) \equiv\left((\forall t) \operatorname{start}(s)<t \supset\left(\exists s^{\prime}\right) s \prec s^{\prime} \wedge t<\operatorname{start}\left(s^{\prime}\right) \wedge \operatorname{proper}\left(s^{\prime}\right)\right)
$$

Thus, the definition of legal is done in terms of proper, which, in turn, is defined in terms of legal ${ }_{\text {poss }}$. Therefore, we concentrate our interest on the subtree in which all the situations are legal with respect to the action preconditions. Notice that according to (15) for a situation to be legal, at any time $t$ after its start, some action must always be possible some time $t^{\prime}$ after $t$.

In what follows we discuss several forms in which the notion of occurrence might arise in knowledge representation. We divide occurrence statements into several classes. We discuss occurrences that cannot be prevented. We also discuss occurrences that arise unless certain preventing conditions are satisfied. In each of the cases discussed, a simple example is presented. To model each type of occurrence we introduce new occurrence predicates and establish constraints between proper and these predicates.

It should be noted here that the notion of a history or partial path, which is formally equivalent to a situation, is essential in the definition of occurrences. In fact, the basic language introduced in the previous section might be considered as the foundational layer that allows one to represent occurrences. Different notions of occurrences correspond to different uses of the path predicates. Some reader might regard the family of occurrence predicate as too diverse, and might feel that a single notion of occurrence should be defined. Indeed, the definition of hasOccurred for actions, times, and histories (or situations) corresponds to this single occurrence predicate.

\subsection{Non-Preventable Occurrences}

\subsubsection{Occurrences in Time}

Consider the sentence:

The sun will rise tomorrow at 6:03 a.m.

Assuming that this sentence is true, we are faced with the problem of representing the occurrence in the Situation Calculus. In [29], Pinto and Reiter addressed this problem by stating that there is an actual line that describes the real evolution of the world and that in this line the sun rises at 6:03 a.m. Unfortunately, this way of modeling this type of occurrence allows for hypothetical developments in which the sunrise does not happen at all. The problem with Pinto and Reiter's framework is that they consider occurrences as being assertions regarding the actual line rather than assertions about the nature of the world. Instead, we consider occurrences to be assertions that constrain the paths in the tree that are proper. We introduce simple non-preventable occurrences with the predicate occurs $s_{n p} \subseteq \mathcal{A} \times \mathcal{T}$ such that occurs $s_{n p}(a, t)$ will mean that the action must be part of any legal history. 
Thus, we have:

$$
\operatorname{proper}(s) \supset\left[\operatorname{occurs}_{n p}(a, t) \wedge t<\operatorname{start}(s) \supset \text { hasOccurred }(a, t, s)\right] .
$$

Thus, the sunrise statement above would be modeled as:

$$
\text { occurs }_{n p}(\text { Sunrise, 6:03am). }
$$

Now we have:

Theorem 3.1 From axioms (1) to (16) it follows that any legal situation that starts after a time must include all actions that non-preventably occur before time $t$ :

$$
\operatorname{occurs}_{n p}(a, t) \wedge \text { legal }(s) \wedge t<\operatorname{start}(s) \supset \text { hasOccurred }(a, t, s) .
$$

PROOF: It follows directly from (15) and (16).

As illustrated by the example of section 4.1, theorem 3.1 establishes essential conditions to deal with narratives.

\subsubsection{Triggered Occurrences}

A triggered occurrence is related to the notion of triggers in active database systems [38]. A trigger is a relationship between two actions. If $a_{1}$ and $a_{2}$ are actions, then $a_{1}$ triggers $a_{2}$ if the first action causes the second one to occur at a later time. It is not our intention to provide an account for causality. However, the formal language that we have introduced provides the necessary support for modeling crucial features of causation ${ }^{1}$.

As a first example consider:

If you eat the forbidden fruit you will be expelled.

In this example, we are given information that is counterfactual in nature. We are told that if we were to do something, then something else would happen as well. One attempt at modeling this piece of information might $b^{2}$ :

$$
\operatorname{occurs}_{\mathrm{np}}(\text { EatFruit, } \mathrm{t}) \supset \operatorname{occurs}_{\mathrm{np}}(\text { Expel }, t+\Delta) \text {. }
$$

Unfortunately, this is inadequate. The reason is that if occurs $\mathrm{np}_{\mathrm{np}}$ (EatFruit, $\mathrm{t}$ ) is not provable, we would not be able to correctly hypothesize about the consequences that eating forbidden fruits might have. This is because it is not possible to treat material implication as counterfactual implication. In order to model this type of occurrence statements, we introduce the predicate occurs $_{\text {to }} \subseteq \mathcal{A} \times \mathcal{T} \times \mathcal{S}$. The literal occurs to $_{o}(a, t, s)$ is intended to mean that action $a$ will definitely occur at time $t$ after $s$ has started. Therefore, to model the previous sentence, we write:

$$
\text { EatFruit } \in c \supset \text { occurs }_{t o}(\text { Expel, start }(d o(c, s))+\Delta, \operatorname{do}(c, s))
$$

\footnotetext{
${ }^{1}$ In particular, causal relations between occurring events, as opposed to causal effects of actions on fluents.

${ }^{2}$ We use teletype font to indicate that we consider the sentence to incorrectly model the knowledge we want to express.
} 
Thus, if $c$ is some concurrent action that includes an EatFruit primitive action, then the Expel action will necessarily occur $\Delta$ time units later. For a more earthly example, let $A_{1}$ denote the event "the high temperature alarm goes off" and $A_{2}$ denote the action "initiate emergency cool-down procedure". Then, to specify that $A_{1}$ triggers $A_{2}$ after $\Delta$ time units, we should write:

$$
A_{1} \in c \supset \text { occurs }_{t o}\left(A_{2}, \operatorname{start}(\operatorname{do}(c, s))+\Delta, \operatorname{do}(c, s)\right)
$$

Since trigger statements have a common syntactical form, we can introduce the predicate trigger $\subseteq \mathcal{A} \times \mathcal{A} \times \mathcal{T}$ as an abbreviation:

$$
\text { trigger }\left(a_{1}, a_{2}, \delta\right) \equiv\left[(\forall c, s) a_{1} \in c \supset \text { occurs }_{t o}\left(a_{2}, \operatorname{start}(\operatorname{do}(c, s))+\delta, \operatorname{do}(c, s)\right)\right]
$$

This notion of trigger can be used to model some physical phenomena at an abstract level. For instance, we can write trigger (Drop, HitGround, $\Delta$ ) to mean that $\Delta$ time units after a Drop is performed a HitGround event occurs. Thus, triggers allow us to model natural events at a coarse level of granularity, abstracting away from details of the physical processes involved. There are circumstances in which we might prefer to reason about natural actions $[24,25,34]$ in this manner, as opposed to treating them as a result of some intricate physical process.

The set of situations in proper are further constrained by occurs $s_{t}$. In fact, we need to introduce a new necessary condition for this predicate, namely:

$$
\operatorname{proper}(s) \supset\left[\operatorname{occurs}_{t o}\left(a, t, s^{\prime}\right) \wedge t<\operatorname{start}(s) \wedge s^{\prime} \prec s \supset \text { hasOccurred }(a, t, s)\right] \text {. }
$$

Theorem 3.2 From axioms (1) to (18) it follows that if $a_{1}$ is an action that triggers $a_{2}$, then $a_{2}$ will be triggered in all legal developments:

$$
\begin{array}{r}
\text { occurs }_{n p}\left(a_{1}, t\right) \wedge \text { trigger }\left(a_{1}, a_{2}, \delta\right) \wedge \text { legal }(s) \wedge t+\delta<\operatorname{start}(s) \supset \\
\text { hasOccurred }\left(a_{2}, t+\delta, s\right) .
\end{array}
$$

PROOF: It follows from (10), (11), (16), (17) and (18).

It can be argued that triggered occurrences do not arise in the real world. Indeed, events or actions are normally triggered by conditions that are true in the world. For instance, in the fruit eating example, one might argue that the Expel action is triggered by the condition resulting from Eat (i.e., Fruit - eaten) and not from the Eat action itself. While this seems a reasonable view for modeling the world as it really is, the triggering events concept is a useful abstraction. In particular, when modeling or specifying artificial devices, one would like to express relations between actions in this manner. For instance, this is true of control systems, or active databases, where one wants systems to react to events happening in the world (alarms going off, transactions being attempted, etc.). Therefore, triggers need to be supported in order to allow for a higher level of abstraction.

\subsection{Conditional Occurrences}

\subsubsection{Preventable Occurrences.}

Consider the following assertion "the train to Ottawa leaves every day at 7 p.m." To simplify things, we will assume that this information is valid forever (i.e., the train schedule does not 
change). However, this piece of knowledge must not be taken literally, even if we consider that it is valid forever. In fact, this statement is defeasible in the sense that the train will leave as long as nothing wrong happens. Knowledge of this kind is very common and we will refer to this type of occurrence as a preventable occurrence. An occurrence is said to be preventable if it depends on the state of the world at the time the occurrence has been predicted.

We introduce a new special predicate occurs $s_{p} \subseteq \mathcal{A} \times \mathcal{T}$ to state that a given action occurs if it is possible for it to occur. For instance:

$$
\psi(t) \supset \text { occurs }_{p o}(\text { TrainLeaves }, t)
$$

states that every time that $\psi$ is true of a time point $t^{3}$, the train leaves if it is possible for it to leave.

The relationship between occurs $s_{p o}$ and the predicate proper is established with a new necessary condition for propoer situations:

$$
\begin{aligned}
\operatorname{proper}(s) \supset\left[\operatorname{occurs}_{p o}(a, t)\right. & \wedge t<\operatorname{start}(s) \wedge \\
\operatorname{Sit}\left(t, s, s_{h}\right) & \wedge \operatorname{Poss}(a, s) \supset \operatorname{hasOccurred}(a, t, s)] .
\end{aligned}
$$

Assume that an action $a$ is said to preventably occur at time $t$. Then, given this new constraint on proper histories, any situation $s$ that starts after $t$ must include $a$ in its history, unless $a$ was not possible at time $t$ along $s$ 's history.

\subsubsection{Conditional Triggered Occurrences}

Consider the assertion "If my neighbor's burglar alarm goes off while I am at home, I will call the police." The difference between a conditional trigger and a non-preventable one is that the caused action can be averted. Here, we introduce the predicate occurs $s_{c t} \subseteq \mathcal{A} \times \mathcal{T} \times \mathcal{S}$ in order to model this type of trigger. The literal occurs $s_{c t}(a, t, s)$ is interpreted to mean that action $a$ will occur at time $t$ after situation $s$, unless it is not possible for it to occur.

The example of the alarm is handled as:

$$
\text { AlarmOff } \in c \supset \text { occurs }_{c t}(\text { CallPolice, } \operatorname{start}(d o(c, s))+\Delta, \operatorname{do}(c, s))
$$

Thus, I will call the police $\Delta$ time units after the alarm goes off, unless I am prevented from doing so. The new necessary condition for proper becomes:

$$
\begin{aligned}
\operatorname{proper}\left(s_{h}\right) \supset \operatorname{occurs}_{c t}\left(a, t, s_{1}\right) \wedge t<\operatorname{start}\left(s_{h}\right) \wedge \\
s_{1} \prec s_{h} \wedge \operatorname{Sit}\left(t, s_{t}, s_{h}\right) \wedge \operatorname{Poss}\left(a, s_{t}\right) \supset \operatorname{hasOccurred}\left(a, t, s_{h}\right) .
\end{aligned}
$$

Formula (20) is analogous to (18) with the added condition that the occurring action be possible when it is meant to occur.

\footnotetext{
${ }^{3} \psi$ might be a formula like $(\exists i) t=T_{0}+i \times K$, where $K$ and $T_{0}$ are some positive constants and $i$ denotes a positive integer number.
} 


\subsection{Simple Occurrences}

In some cases we might want to express knowledge regarding some action occurrence without specifying any details. For instance, we might want to state that action Finish will eventually occur; i.e., that the action will appear somewhere in the time line. If we were using some linear time logic we would express this with a sentence like:

$$
(\exists \mathrm{t}) \operatorname{occurs}(\text { Finish, } \mathrm{t})
$$

which might be taken to mean that eventually the action Finish will occur. In a branching time logic, we need to specify that Finish must arise in any possible development. In our language, we could choose to model the eventual occurrence of Finish with the statement:

$$
(\exists \mathrm{t}) \operatorname{occurs}_{\mathrm{np}}(\text { Finish, } \mathrm{t}) .
$$

Indeed, this statement ensures that there is a time $t$ such that any legal situation that starts after $t$ has a history that includes Finish. Unfortunately, (so**) is too strong. The problem that arises is that this statement forces Finish to occur at the same time in all the histories. What we need is a statement that leaves the time of the occurrence of Finish unconstrained. To achieve this, we introduce the predicate occurs $_{b y} \subseteq \mathcal{A} \times \mathcal{T}$. The intended meaning of $\operatorname{occurs}_{b y}(a, t)$ is that action $a$ has occurred before time $t$. Thus, instead of ( so $\left.^{*}\right)$ we write ${ }^{4}$ :

$$
(\exists t) \operatorname{occurs}_{b y}(a, t)
$$

which constrains proper as follows:

$$
\operatorname{proper}(s) \supset\left[\operatorname{occurs}_{b y}(a, t) \wedge t<\operatorname{start}(s) \supset\left(\exists t^{\prime}\right) t^{\prime}<t \wedge \operatorname{hasOccurred}\left(a, t^{\prime}, s\right)\right] .
$$

Therefore, the statement occurs $b y$ (Finish, $t$ ) states that all the legal situations that start at $t$ or later have histories in which Finish has already taken place.

The simple occurrences discussed here are essential to express statements like "Eventually $a$ occurs". For instance, "after getting into Dr. Jec's office, I will eventually come out." Statements of the form (so) allow us to express the future occurrence of actions without over-committing to a time at which they ought to occur. This notion of eventuality is related to the similar notion in temporal logics [15]. The main difference is that, in temporal logics, the operator $\square$, used to express the notion of eventuality, is applicable to truth valued sentences as opposed to actions or events.

\subsection{Final Remarks on Proper Histories}

As mentioned before, we consider proper to be a defined predicate whose definition is obtained from the closure of its necessary conditions. Therefore, by closing the axioms for predicate proper with (14), (16), (18), (19), (20) and (21), we obtain:

\footnotetext{
${ }^{4}$ The reader should keep in mind that the sentences (so*) and (so), which appear almost identical, belong to theories with a totally different background axiomatization.
} 


$$
\begin{aligned}
\text { proper }_{(s) \equiv} & \text { legal }_{\text {poss }}(s) \wedge \\
& {\left[\text { occurs }_{n p}(a, t) \wedge t<\operatorname{start}(s) \supset \text { hasOccurred }(a, t, s)\right] \wedge } \\
& {\left[\text { occurs }_{t o}\left(a, t, s^{\prime}\right) \wedge t<\operatorname{start}(s) \wedge s^{\prime} \prec s \supset \text { hasOccurred }(a, t, s)\right] \wedge } \\
& {\left[\text { occurs }_{p o}(a, t) \wedge t<\operatorname{start}(s) \wedge\right.} \\
& \left.\operatorname{Sit}\left(t, s, s_{h}\right) \wedge \operatorname{Poss}(a, s) \supset \operatorname{hasOccurred}(a, t, s)\right] \wedge \\
& {\left[\text { occurs }_{c t}\left(a, t, s_{1}\right) \wedge t<\operatorname{start}\left(s_{h}\right) \wedge\right.} \\
& \left.s_{1} \prec s_{h} \wedge \operatorname{Sit}\left(t, s_{t}, s_{h}\right) \wedge \operatorname{Poss}\left(a, s_{t}\right) \supset \operatorname{hasOccurred}\left(a, t, s_{h}\right)\right] \wedge \\
& {\left[\text { occurs }_{b y}(a, t) \wedge t<\operatorname{start}(s) \supset\left(\exists t^{\prime}\right) t^{\prime}<t \wedge \operatorname{hasOccurred}\left(a, t^{\prime}, s\right)\right] . }
\end{aligned}
$$

Thus, the definition of proper is done in terms of the occurrence predicates, which are primitive, and should be specified by the axiom writer. We do not claim that the set of occurrence predicates that we have presented is exhaustive. In fact, there are other types of occurrence that can also be specified in a similar form. For instance, we could add simple occurrences that are preventable and/or triggered. The main contribution of this work is to set the stage for the definition of these notions of occurrences. The closure of this predicate should not be taken as a completeness assumption. Rather, it corresponds to a complete specification of the set of proper situations.

\section{Applications}

In this section, we take $\Sigma$ to denote the background axioms, i.e.:

- The basic axioms of the Situation Calculus, (1)-(9).

- Definition of hasOccurred plus the path predicates, (10)-(13).

- Definition of the legal predicate (15), the definition of the notion of trigger (17), and the definition of proper history (22).

\subsection{Narratives and Preferred Developments}

\subsubsection{Narratives}

Consider a slightly modified version of Miller and Shanahan's briefcase example[22]:

Before breakfast one morning, a lecturer, Mary, puts her lecture notes inside the briefcase. She eats breakfast, and shortly thereafter carries her briefcase to college. Mary concludes that the lecture notes are in her briefcase at college. However, shortly after she sits down at her desk, her husband telephones to apologize for accidentally removing her notes from the briefcase before she left for work. With her new knowledge of her husband's actions, Mary concludes that her notes are not at college.

We will consider the actions PutIn, Eat, TakeNotes, Carry which denote the put in briefcase, eat breakfast, take notes from briefcase and carry briefcase to college actions.

Let $\Omega$ denote the following sentences: 


$$
\begin{aligned}
& \operatorname{occurs}_{n p}(\text { Put In, 6:30am), } \\
& \operatorname{occurs}_{n p}(\text { Eat, 6:35am), } \\
& \text { occurs }_{n p}(\text { Carry, 7:00am }) .
\end{aligned}
$$

Then, using theorem 3.1 we obtain:

Observation 4.1 The background axioms $\Sigma$, along with the occurrence statements $\Omega$, entail that the history of any legal situation, that starts after the time of the last action in the narrative, will contain all the actions in the narrative.

$$
\begin{aligned}
\Sigma \cup \Omega \vDash(\forall s) \text {.legal }(s) \wedge 7 & : 00 a m<\operatorname{start}(s) \supset \\
& \text { hasOccurred }(\text { Put In, 6:30am, } s) \wedge \\
& \text { hasOccurred }(\text { Eat }, 6: 35 a m, s) \wedge \\
& \text { hasOccurred }(\text { Carry, 7:00am, } s) .
\end{aligned}
$$

PROOF: It follows directly from the definition of legal (15), and constraint (16).

Thus, the question is whether we can prove that Mary's notes are at College with her, given the set $\Omega$ of occurrences. According to the actual line of events ${ }^{5}$, this should be the case. However, what should a Situation Calculus based theory entail? If we want to keep the hypothetical view of the world we must accept the possibility that other things might happen aside from the occurrences that are explicitly stated. For instance, it might be the case that the action TakeNotes, that refers to the husband's action, happens or that it does not. Since our theory does not say that it occurs, then it might or it might not occur.

In fact, we have:

Observation 4.2 Given axioms $\Sigma \cup \Omega$, there are legal histories that contain the action Take Notes by which Mary's husband removes the notes from her briefcase. This can be formalized:

$$
\Sigma \cup \Omega \vDash(\exists s, t) \text {.legal }(s) \wedge \text { 7:00am<start }(s) \wedge \text { hasOccurred }(\text { TakeNotes }, t, s)] .
$$

Each path in the tree of situations can be seen as a possible way in which the world might evolve. This is akin to Kripke's possible worlds semantics. That is, each one of the paths represents a possible world or a possible development. If we state that some action $A$ occurs, then we mean that any path that does not contain $A$ is deemed ill-formed. Also, in a similar spirit to the possible worlds semantics, the negation of an occurs literal will not mean that its action argument does not appear in any path, it simply means that there is a hypothetical course of events in which this action is not present.

\subsubsection{Preferred Developments}

A great advantage of the Situation Calculus over modal logics is that situations, which could be viewed as worlds, are objects in the language. Therefore, we can predicate over situations

\footnotetext{
${ }^{5}$ Here, we use the terms event and action interchangeably
} 
within the language. Here, we will use this feature of the Situation Calculus to define a notion of preference between situations. The idea is to be able to compare two legal situations and decide which one might better explain the information that we have at hand. The preference relation between two situations will be based on a comparison between their common histories. Thus, if $s$ starts at time 12 and $s^{\prime}$ starts at time 15 , then we compare the actions that appear in their histories between times 0 (the start of $S_{0}$ ) and time 12 (the situation that starts earlier). The preference that we define below is such that $s$ is preferred over $s^{\prime}$, written $s \unlhd s^{\prime}$, if all the actions that appear in $s$ between time 0 and time $\min \left(\operatorname{start}(s), \operatorname{start}\left(s^{\prime}\right)\right)^{6}$ also appear in $s^{\prime}$. The definition is:

$$
\begin{aligned}
& \operatorname{legal}(s) \wedge \text { legal }\left(s^{\prime}\right) \supset\left(s \unlhd s^{\prime} \equiv\right. \\
& (\forall a, t)\left[t \leq \min \left(\operatorname{start}(s), \operatorname{start}\left(s^{\prime}\right)\right) \wedge \operatorname{hasOccurred}(a, t, s) \supset\right. \\
& \text { hasOccurred } \left.\left.\left(a, t, s^{\prime}\right)\right]\right) .
\end{aligned}
$$

Thus, if one looks at the narrative formalized with sentences (23)-(25), we have that the situation $\operatorname{do}\left([\{\text { Put In }\},\{\text { Eat }\},\{\text { Carry }\}], S_{0}\right)^{7}$, with time assignments:

$$
\begin{aligned}
& \operatorname{start}\left(S_{0}\right)=0, \\
& \operatorname{start}\left(\operatorname{do}\left(\{\text { PutIn }\}, S_{0}\right)\right)=6: 30 a m, \\
& \left.\operatorname{start}\left(\operatorname{do}\left([\{\text { Put In }\},\{\text { Eat }\}], S_{0}\right)\right)\right)=6: 35 a m, \\
& \operatorname{start}\left(\operatorname{do}\left([\{\text { Put In }\},\{\text { Eat }\},\{\text { Carry }\}], S_{0}\right)\right)=7: 00 a m,
\end{aligned}
$$

would be preferred over the legal situation $\operatorname{do}\left([\{\right.$ PutIn $\},\{$ Eat $\},\{$ TakeNotes $\},\{$ Carry $\left.\}], S_{0}\right)$, with time assignments:

$$
\begin{aligned}
& \operatorname{start}\left(S_{0}\right)=0, \\
& \operatorname{start}\left(\operatorname{doo}\left([\{\text { PutIn }\}], S_{0}\right)\right)=6: 30 \text { am, } \\
& \operatorname{start}\left(\operatorname{do}\left([\{\text { Put In }\},\{\text { Eat }\}], S_{0}\right)\right)=6: 35 \text { am, } \\
& \operatorname{start}\left(\operatorname{do}\left(\left[\{\text { Put In }\},\{\text { Eat }\},\{\text { TakeNotes }\}, S_{0}\right]\right)\right)=6: 40 \text { am, } \\
& \operatorname{start}\left(\operatorname{do}\left([\{\text { PutIn }\},\{\text { Eat }\},\{\text { TakeNotes }\},\{\text { Carry }\}], S_{0}\right)\right)=7: 00 \text { am. }
\end{aligned}
$$

Also, we can define:

$$
\operatorname{minimal}(s) \equiv \operatorname{legal}(s) \wedge\left(\forall s^{\prime}\right)\left[\left(\operatorname{legal}\left(s^{\prime}\right) \wedge s^{\prime} \unlhd s\right) \supset s \unlhd s^{\prime}\right],
$$

where minimal is true of a situation iff it is a most preferred situation.

Using these predicates, we have:

Observation 4.3 Let $\Sigma_{m}$ be the definitions (27) and (30). We have that:

$$
\begin{aligned}
\Sigma \cup \Omega \cup \Sigma_{m} \vDash(\forall s) \text { legal }(s) & \wedge \text { 7:00am }<\operatorname{start}(s) \wedge \operatorname{minimal}(s) \supset \\
& \neg(\exists t) t \leq 7: 00 a m \wedge \text { hasOccurred }(\text { TakeNotes }, t, s)] .
\end{aligned}
$$

PROOF: From observation 4.1 we know that all legal situations that start after the Carry action will include the three actions explicitly mentioned in the narrative. Thus, if $s^{\prime}$ were a legal situation that included a TakeNotes action before 7:00 am, there would always be a preferred situation not including it. Therefore, $s^{\prime}$ would not be minimal.

\footnotetext{
${ }^{6}$ We take $\min \left(r_{1}, r_{2}\right)$ denote the minimum real between $r_{1}$ and $r_{2}$.

${ }^{7}$ The function symbol do takes a concurrent action as a fi rst argument. The set $\{P u t I n\}$ is used to denote a concurrent action composed of a single action.
} 
Thus, with our approach to handling occurrences, we are still able to do all sorts of hypothetical reasoning. But, more interestingly, we can also define preference criteria for the set of legal situations. As part of our ongoing work, we plan to explore the issue of defining a richer set of properties to describe situations. For instance, given some notion of elementary likelihood, perhaps defined in terms of agent choices, one can define a derived notion of situation likelihood. In such a language, one would be able to describe situations that are likely to arise. Given this notion of likelihood, one can devise planning systems that plan for likely courses of events first and reason about possible less likely developments later.

\subsubsection{Narratives and Planning}

One advantage of our approach to representing occurrences is the fact that planning can be specified as a deductive problem within a first order framework. Following Green [5], a planning problem corresponds to the question "Does there exist a situation $s$ that satisfies a goal description $G$ ?" If we assume that there are no occurrence specifications, in a Situation Calculus language we would like to find a constructive proof for the following theorem:

$$
\Sigma \vDash(\exists s) G(s) .
$$

Thus, finding a situation $S$ for which $G(S)$ is true solves the planning problem. This solution is satisfactory because the proof would yield the specification of a situation as a sequence of actions performed in $S_{0}$ as:

$$
S=d o\left(\left[c_{1}, c_{2}, \ldots, c_{p}\right], S_{0}\right),
$$

where $p$ is the number of actions in the plan. Therefore, the theorem guarantees that after performing $c_{1}, c_{2}, \ldots, c_{p}$ we would reach a situation in which the goal is true. Applying the same idea to theories in the Situation Calculus language presented in this article, a planning problem is formulated as finding a constructive proof for the theorem:

$$
\Sigma \cup \Omega \vDash(\exists s) G(s) .
$$

Where $\Omega$ denotes a set of occurrence statements. Therefore, any situation that is found to conform to the goal specification would also conform to the occurrence constraints.

For instance, in the spirit of the previous example, let us assume that Mary's husband wants to play a tasteless practical joke on her. Thus, he would like a plan that would lead to a situation in which her notes don't make it to college with her. Using the definition (31) we need to specify a goal formula that characterizes the husband's goal. A first requirement is that the situation obtained be legal and that Mary's notes don't make it to school:

$$
g_{o}(s) \equiv \operatorname{legal}(s) \wedge \neg \text { notesAtSchool }(s),
$$

where notesAtSchool is a fluent predicate that is true in those situations in which Mary's notes are at school. Finally, the goal $G$ can be defined as:

$$
\left.G(s) \equiv g_{0}(s) \wedge\left(\forall s^{\prime}\right)\left[g_{0}\left(s^{\prime}\right) \wedge s^{\prime} \unlhd s\right) \supset s \unlhd s^{\prime}\right],
$$

Thus, in this case, the plan is formulated as one in which a minimality criterion, like the one defined in (30), is also imposed. That is, the plan that we want is obtained by making the explicit assumption that the the agent arrives at a goal situation by performing a minimal number of actions. 


\subsection{Scheduled Events}

In this subsection we consider the following type of situation: An action $A_{1}$ triggers $A_{2}$, which would occur, if possible, $\Delta$ time units after $A_{1}$. Action $A_{2}$ cannot occur unless it has been triggered by $A_{1}$. This type of situation is common when modeling the intended behavior of certain industrial processes. For instance, every time we receive a request, we schedule an acknowledge event. The difference between the triggers we discussed previously and these scheduled events is that the latter are used to model triggered events that cannot occur unless a specific action triggers it.

To model this we introduce a fluent predicate $S$ cheduled $\subseteq \mathcal{A} \times \mathcal{T} \times \mathcal{S}$. Initially, we may assume that there are no scheduled actions:

$$
\neg \text { Scheduled }\left(a, t, S_{0}\right)
$$

Also, for $A_{2}$ we have:

$$
\operatorname{Poss}\left(A_{2}, s\right) \equiv(\exists \delta) \delta>0 \wedge \text { Scheduled }\left(A_{2}, \delta, s\right) \wedge \pi_{A_{2}}(s)
$$

Thus, $A_{2}$ can only occur if it has been scheduled. $\pi_{A_{2}}(s)$ are further preconditions for the performance of $A_{2}$ in situation $s$. To complete the formalization, we need to add that:

$$
\begin{aligned}
& \text { trigger }\left(A_{1}, A_{2}, \Delta\right), \\
& A_{1} \in c \supset \operatorname{Scheduled}\left(A_{2}, \Delta, \operatorname{do}(c, s)\right) .
\end{aligned}
$$

As a more specific example, consider the following information:

My house has a burglar alarm. If the alarm is connected, I have exactly 60 seconds to deactivate it after opening the main door. If I am unable to disconnect the alarm, it will go off ${ }^{8}$.

The axioms for this example are:

$$
\begin{aligned}
& \neg \text { Scheduled }\left(a, \delta, S_{0}\right), \\
& \text { Poss }(\text { AlarmOff }, s) \equiv \text { Scheduled }(\text { AlarmOff, } \delta, s) \wedge \text { alarmConnected }(s), \\
& \text { trigger }(\text { OpenDoor, AlarmOff, } 60), \\
& \text { Scheduled }(\text { AlarmOff, } 60, \operatorname{do}(\{\text { OpenDoor }\}, s)) .
\end{aligned}
$$

Therefore, if the door is open, then (38) ensures that the alarm will be scheduled to go off 60 seconds after, unless the alarm is not connected at that time. To complete the formalization of Scheduled we need a successor state axiom[31]. This axiom is trivial in this case, since there are no actions that change the value of the fluent Scheduled.

\subsection{Active Databases}

The Situation Calculus has proven to be an extremely powerful language. Here, we argue that the language can be used effectively as a tool for the modeling and analysis of active database systems. As an illustration, consider the following example (proposed by Zaniolo [39]), where there are two relations in a relational database EMP (E\#, Ename, JobTitle, SAL, Dept\#) and HPaid (JobTitle):

\footnotetext{
${ }^{8}$ Thanks to Michael Gruninger for suggesting this example.
} 
Upon an insertion into EMP or an update to EMP, the new SAL is checked, and if it exceeds $\$ 100,000$, then the JobTitle of this employee is added to HPaid, assuming that it was not there already.

This is straightforward to model using the language that we have described already. Indeed, following Reiter [32], each database relation is modeled as a fluent. For simplicity, we eliminate fields in the relations that are not relevant for the example and assume that the language is conveniently extended with subsorts of the sort $\mathcal{D}^{9}$. We use the fluent $e m p$ that takes an employee number, a job-title, a salary and a situation as arguments. Thus, $\operatorname{emp}(e \#, j t, s a l, s)$ is true in the situation $s$ if the employee with number $e \#$ has a job with title $j t$ and salary sal. Furthermore, we introduce the fluent $H$ Paid that takes a job title and a situation as arguments. Thus, $H P$ aid $(j t, s)$ is true in $s$ if the job title $j t$ is highly paid in that situation. Also, we have the actions upgradeSalary (e\#, newsal) and addJobTitle (jt). The first action is meant to change the salary of the employee with number $e \#$ to a new salary newsal. The second action is used to add a new job title to the table of highly paid positions.

We assume that the action upgradeSalary has no preconditions. Also, we assume that addJobTitle is possible if and only if the job title is not already in the table HPaid. This is written as:

$$
\begin{aligned}
& \text { Poss }(\text { upgradeSalary }(e \#, \text { newsal }), s) \equiv \text { True, } \\
& \operatorname{Poss}(\operatorname{addJobTitle}(j t), s) \equiv \neg H \operatorname{Paid}(j t, s) .
\end{aligned}
$$

As a next step, we present successor state axioms that tell us when the fluents are true in a situation $d o(c, s)$. For fluent $e m p$, we have:

$$
\begin{aligned}
& \operatorname{Poss}(c, s) \supset \operatorname{emp}(e \#, j t, \text { sal }, \text { do }(c, s)) \equiv \\
& \qquad m p(e \#, j t, \text { sal }, s) \wedge \text { upgradeSalary }(e \#, \text { newsal }) \notin c \vee \\
& \text { upgradeSalary }(e \#, \text { sal }) \in c .
\end{aligned}
$$

On the other hand, for HPaid, we have:

$$
\begin{aligned}
& \operatorname{Poss}(c, s) \supset \operatorname{HPaid}(j t, d o(c, s)) \equiv \\
& H \operatorname{Paid}(j t, s) \vee \operatorname{addJobTitle}(j t) \in c .
\end{aligned}
$$

Finally, in order to model the trigger of an addJobTitle event by the upgradeSalary event provided that the salary is over the $\$ 100,000$ threshold, we have:

$$
\begin{array}{r}
{[\text { newsal }>\$ 100,000 \wedge e m p(e \#, j t, \text { sal }, s) \wedge \text { upgradeSalary }(e \#, \text { newsal }) \in c] \supset} \\
\operatorname{occurs}_{c t}(\operatorname{addJobTitle}(j t), \operatorname{start}(\operatorname{do}(c, s))+\Delta, \operatorname{do}(c, s)),
\end{array}
$$

where $\Delta$ is some positive real constant.

Notice the similarity of this formula with formula (17) that defines the notion of a trigger. The reader might wonder why on this occasion we did not use definition (17), or a similar definition involving conditional triggers (see section (3.3.2)). The reason is that formula (17) defines triggers as context independent. In fact, the trigger predicate does not include a situation argument. In this example, however, the triggered event is context dependent. In fact, the triggered

\footnotetext{
${ }^{9}$ To be completely rigorous we need to add sorts for employee numbers, job titles, and other domain specifi c sorts.
} 
action is addJobTitle $(j t)$, and the argument $j t$ is obtained from the context in which the triggering action is executed.

Another important detail has to do with the value of the constant $\Delta$. An appropriate value for this example might be a small constant that guarantees that the transaction is performed immediately after or soon after the triggering event occurs. In some applications, one might need to ensure that no transaction can possibly be performed while a triggered event is not completed. This can be accommodated by adding a fluent identifying pending transactions, so that that no transactions, other than the pending ones, are possible until there are no pending transactions.

It should be clear that the axioms above guarantee that $\Delta$ units of time after an upgradeSalary is performed, an addJobTitle is also performed.

\section{Relationship with other Approaches}

\subsection{Linear Time Approaches in the Situation Calculus}

As mentioned before, previous approaches to handle occurrences and narratives in the Situation Calculus relied on the idea of imposing a time line as one path in the Situation Calculus tree. One of this approaches is Pinto and Reiter's actual line approach. Note that the formalism of Pinto and Reiter does not deal with concurrent actions. However, in [24] Pinto and Reiter's work is extended to deal with concurrent actions. A minor variation of this formalism is used in this section. Our intent is to show that the approach presented in this paper is a generalization of the actual line approach.

Assume that we have the following axiomatization for actual (based on [28]):

$$
\begin{aligned}
& \operatorname{actual}\left(S_{0}\right), \\
& (\forall c, s) \cdot \operatorname{actual}(\operatorname{do}(c, s)) \supset \operatorname{actual}(s) \wedge \operatorname{Poss}(c, s), \\
& \left(\forall c_{1}, c_{2}, s\right) \cdot \operatorname{actual}\left(\operatorname{do}\left(c_{1}, s\right)\right) \wedge \operatorname{actual}\left(\operatorname{do}\left(c_{2}, s\right)\right) \supset c_{1}=c_{2} .
\end{aligned}
$$

Furthermore, we have the predicate occurs $\mathcal{T}$ which is true for an action and a time point if the action occurs at the specified time point:

$$
\operatorname{occurs}_{\mathcal{T}}(a, t) \equiv(\exists c, s) . a \in c \wedge \operatorname{actual}(\operatorname{do}(c, s)) \wedge \operatorname{start}(\operatorname{do}(c, s))=t .
$$

Assume that a narrative is represented using a set $\Omega$ of occurs $_{n p}$ literals. To establish a relationship between our new approach to occurrences and the actual line approach, we add:

$$
\operatorname{occurs}_{n p}(a, t) \supset \operatorname{occurs}_{\mathcal{T}}(a, t) .
$$

Given this we have:

Theorem 5.1 Let $\Sigma_{a}$ denote axioms of (1)-(16), (22) and (39)-(43). Also, let $\Omega$ denote a narrative expressed as occurs $s_{n p}$ literals. From Sigma $\cup \Omega$ we can infer that the actual line of situations must be legal. Thus:

$$
\Sigma_{a} \cup \Omega \vDash(\forall s) \operatorname{actual}(s) \supset \operatorname{legal}(s)
$$

PROOF: Follows from the definitions. Intuitively, (43) forces the actual line to conform to the legality as defined by axiom (15). 
Theorem 5.2 Let $\mathcal{M}$ be a model of $\Sigma_{a}$ (as defined above). Also, let $\sigma_{\mathcal{S}}[\cdot]$ be an assignment function for situation variables. The following holds:

If

$$
\mathcal{M}, \sigma_{\mathcal{S}}[s] \vDash \operatorname{legal}(s)
$$

then, there is a model $\mathcal{M}^{\prime}$ that interprets everything the same except for actual and occurs $\mathcal{T}$, such that:

$$
\mathcal{M}^{\prime}, \sigma[s] \vDash \operatorname{actual}(s) \text {. }
$$

PROOF: The proof is done by building an interpretation $\mathcal{M}^{\prime}$ just like $\mathcal{M}$, except for the interpretation of actual. The interpretation of actual in $\mathcal{M}^{\prime}$ can be set in such a way that $\sigma_{\mathcal{S}}(s)$ is actual. Given that $\sigma_{\mathcal{S}}(s)$ belongs to the interpretation of legal it must be the case that $\mathcal{M}^{\prime}$ satisfies the axioms for actual.

An interesting consequence of this theorem is that our theory is categorical for actual if and only if there is a unique legal path in the situation tree. This theorem allow us to claim that our new approach to occurrences subsumes Pinto and Reiter's approach. In fact, a consequence of the theorem is that if an action can be proven to occurs $\mathcal{T}$ at a certain time point, then this action must be present in every legal history that includes that time point. As discussed in the introduction, Shanahan showed that there is an equivalence, under certain conditions ${ }^{10}$, between Miller and Shanahan's characterization of a time line and Pinto and Reiter's. Therefore, under the same conditions, our new approach to occurrences should also subsume Miller and Shanahan's approach.

\subsection{Hypothetical Reasoning in Linear Time Theories}

In [21], Rob Miller proposes a novel approach to perform deductive-style planning in the Event Calculus [8]. Miller's work is based on Shanahan's Circumscriptive Event Calculus [36]. The Circumscriptive Event Calculus solves the frame problem by appealing to Circumscription [17, 12]. The language of the Circumscriptive Event Calculus includes sorts for actions, fluents, time-points, and domain objects. Important predicates are Initiates and Terminates which are ternary predicates with actions, fluents and time-points as arguments. These predicates are used in order to write domain axioms to express effects that actions have on fluents, when the actions are performed at certain time points. If a fluent holds before a time point and an action terminates it at that point, then the fluent will not hold after, unless another action initiates it at a later point. Also, a binary predicate Happens for action and time points is used to mean that some action happens at some time point. A full description of the language would take us too far afield and is not necessary for our present discussion.

At the core of Miller's proposal is the introduction of a distinction between actions that occur, and that are external the agent being modeled, and the actions that are performed by the agent. Thus, Miller introduces the axiom:

$$
\text { Happens }(a, t) \equiv[\operatorname{Occurs}(a, t) \vee \operatorname{Perform}(a, t)] .
$$

\footnotetext{
${ }^{10}$ The conditions refer to the form in which circumscription axioms are applied in both formalizations. The details are beyond the scope of this paper, and the reader is referred to [37, chapter 9] for further details.
} 
The domain axioms would only include specifications for narratives expressed in terms of occurrences. Miller proceeds to specify a circumscription policy that involves the separate minimization of the Initiates and Terminates predicates in the context of the domain effect axioms. Also, he minimizes Occurs in the context of the axioms for Narratives. The final circumscription policy involves other minimizations as well. An important feature of the circumscription policy is that it leaves out Happens and Performs. Therefore, the models of a Miller style Event Calculus theory are minimal in the extension of Occurs but leave the interpretation of Performs free.

A plan $\mathcal{P}$ for a time $\tau$, in Miller's framework, is a formula of the form

$$
\operatorname{Perform}(a, t) \equiv\left(\left(a=\alpha_{1} \wedge t=\tau_{1}\right) \vee \ldots \vee\left(a=\alpha_{n} \wedge t=\tau_{n}\right),\right.
$$

where the $\alpha_{i}$ 's $\tau_{i}$ 's are ground terms, such that each $\tau_{i}$ is greater than $\tau$. If $\mathcal{G}$ denotes a goal, then a valid plan $\mathcal{P}$ is such that the theory entails

$$
\mathcal{P} \supset \mathcal{G}
$$

Furthermore, $\mathcal{P}$ must be satisfiable. However, Miller shows that the test for satisfiability is not necessary for theories of a certain general form.

Finally, hypothetical reasoning about possible plans would involve the use of implications like the one above. Thus, if one wants to reason about a possible plan, one needs to look at the models in which that plan is carried out. This works only if the plans that are entertained are satisfiable.

There are two important differences between Miller's approach and the one we have presented in this article, namely:

- Aside from the use of induction, our approach is first order. On the other hand, Miller's approach is circumscriptive. The second order nature of circumscription needs not be a problem since, in some cases, it can translate into some equivalent first order formula. Unfortunately, the use of circumscription leads to the premature minimization problem, discussed in the introduction. Indeed, the minimization of Occurs precludes one from entertaining situations in which unnecessary ${ }^{11}$ events do occur. Although, one can reason hypothetically about the actions that can be performed.

An important advantage of the circumscriptive approach is that the solution to the frame problem is more general and can be applied to theories that include state constraints as well as effect axioms. However, the correctness of solutions based on circumscription is hard to assess.

- We do not need to give a different treatment to actions performed by the agent and actions that occur externally. In fact, minimizing Occurs separately makes it difficult to include occurrences that are triggered by some action performed by the agent. However, this is not a problem if one characterizes triggers as a relation between world properties and triggered actions.

\section{Conclusion}

A great deal of research in knowledge representation about dynamic domains has concentrated on the modeling of the effects that actions have on the properties of the world. In particular, the

\footnotetext{
${ }^{11}$ Unnecessary from the point of view of the axiomatization
} 
frame problem motivated the development of logical frameworks in which certain common-sense assumptions could be formalized. As Shanahan remarks [37], “... like the mind body problem in philosophy, there's no universal agreement as to what would constitute a solution." Furthermore, Shanahan also suggests that "... we are very close to a complete solution to the frame problem." While this might seem contradictory on first reading, the claim is substantiated by the fact that we have at our disposal a rich set of theoretical tools to deal with the problem.

Most solutions to the frame problem involve the use of some sort of minimal model semantics. In contrast, our work is based on a first order solution to the frame problem (due to Raymond Reiter [31]). However, this solution is not completely general and more work is needed in order to extend it to deal with general theories. For instance, we need to extend this solution to domains in which actions have uncertain effects.

However, if we were to assume that the frame problem is solved, then we need to worry about the problem of writing theories of the world in which we take into account the free will of the agents that populate it. We need languages in which one can characterize how these agents behave. This language should allow us to plan for courses of actions that take into account certain common-sense assumptions that we must make in order to plan. If we are unable to make these assumptions, then planning is not possible. For example, my plan to fly to Toronto is reasonable given that I assume that the agents in the world behave according to certain patterns. For instance, I assume that the airplane pilot will not intentionally crash the plane, etc.

While our approach is strictly first order, the formalization of assumptions of agent-behavior might require more expressive languages. A different approach could be based on second-order circumscriptive axiomatizations. For instance, Miller's work, discussed in section 5.2, goes in this direction, Miller is seeking for a balance between what can be constrained or minimized (external occurrences) and what should be allowed to be free (the agent's actions). This research needs to evolve in order for us to provide support for the characterization of the evolution of the world. This characterization will surely involve common-sense assumptions, without which our agents would be paralyzed.

In this article we present a formal framework that allows one to deal with occurrences in a general form. In particular, the specification of action occurrences constrains the histories that can be considered legal in a branching time structure. We are currently investigating the use of this framework to model agent behavior. For instance, statements of the form "If a message is received from some agent, then a reply must be sent to that agent" can be written as triggering statements. As a first experiment, we are modeling agent interaction through communication protocols, where the agents are constrained to react to events with actions specified by the protocol.

Also, the approach presented allows one to reason about hypothetical developments which obey all occurrence constraints imposed by the occurrence axioms. An essential aspect of our approach is that we rely on the notion of history. This notion is equivalent to the notion of chronicle in Drew McDermott's Temporal Logic for Reasoning about Processes and Plans [19]. To sum up, in this paper we:

1. Build upon a first order solution to the frame problem in the Situation Calculus.

2. Extend the language with explicit references to actions in histories.

3. Use histories to define external occurrences.

4. Define different notions of occurrences that can be used to express conditional occurrences, triggers, narratives, etc. 
There are several important extensions to this work that we would like to pursue, namely:

- Observations: That is, we would like to study the effect of adding statements regarding the values of fluents at different points in time.

- Complex occurrences: We would like to consider occurrence statements involving complex actions (actions that involve conditionals, loops, etc.).

- We would like to investigate how to integrate this approach to occurrences with the current work on the GOLOG language[11] being developed at the Cognitive Robotics group in the University of Toronto. Also, it is important to analyze the relationship between the notion of interrupts in GOLOG with our notion of occurrence.

- In this paper we do not address the computational issues of reasoning. It is likely that tasks like plan verification will be difficult. In particular, non-preventable occurrences may introduce difficulties in proving the legality of situations. The reason is that one might be forced to think about the future of a situation in order to determine its legality. This difficulty does not always arise, and we would like to characterize classes of theories for which this reasoning is easy.

- Planning: The theoretical advances that have been made in knowledge representation using the Situation Calculus provide a framework in which planning can be extended to richer application areas. For instance, in [10], Levesque studies the formal specification of planning problems in the presence of sensing in a Situation Calculus extended with the ability to incorporate knowledge.

The work presented here allows for the incorporation of exogenous events, which can be formalized using occurrence statements. Thus, we would like to extend this work by providing a characterization of planning in the presence of events that are external to the agent that is doing the planning. For instance, it would be interesting to be able to come up with a plan for an agent to go to the airport knowing that the bus to the airport leaves for the airport every hour on the hour. However, we should not be able to prove that the plan is successful unless we assume that the bus does not run into problems. A plan, then, should be provably correct only under explicit assumptions regarding the behavior of external agents.

- In [29], it was shown that the Situation Calculus with actual line subsumes the temporal logic of concurrency [4]. We would like to further explore the issue of expressiveness of the Situation Calculus in comparison to the expressiveness found in conditional and other modal logics.

\section{References}

[1] Allen, J. F. Towards a General Theory of Action and Time. Artificial Intelligence 23 (1984), 123-154.

[2] Bertossi, L., Pinto, J., Saez, P., Kapur, D., and Subramaniam, M. Automating Proofs of integrity constraints in situation calculus. In Lecture Notes in Artificial Intelligence: Proceedings of the 9th International Symposium on Methodologies for Intelligent Systems (1996), Z. Pawlak and Z. W. Ras, Eds., Springer Verlag. To appear. 
[3] Gelfond, M., Lifschitz, V., And Rabinov, A. What are the Limitations of the Situation Calculus? In Working Notes, AAAI Spring Symposium Series. Symposium:Logical Formalization of Commonsense Reasoning. (Mar. 1991), pp. 59-69.

[4] Goldblatt, R. Logics of Time and Computation. CSLI, 1987.

[5] Green, C. C. Applications of Theorem Proving to Problem Solving. In Proceedings of the First International Joint Conference on Artificial Intelligence (IJCAI-69) (1969), pp. 219239. Reprinted in Readings in Artificial Intelligence, Webber, B. L. and Nilsson, N. J., editors, Tioga Publishing Co., Los Altos, California, pp. 202-222, 1981.

[6] Kapur, D., And Zhang, H. RRL: A Rewrite Rule Laboratory. In Proc. Ninth International Conference on Automated Deduction (CADE-9), LNCS 310 (1988), no. 310 in LNCS, Springer-Verlag.

[7] Kowalski, R., AND SAdRI, F. The Situation Calculus and Event Calculus Compared. In Proceedings of the International Logic Programming Symposium, Ithaca, New York (November 1994), M. Bruynooghe, Ed., The MIT Press, pp. 539-553.

[8] Kowalski, R., And Sergot, M. A Logic-based Calculus of Events. New Generation Computing 4 (1986), 67-95.

[9] Lespérance, Y., Levesque, H., Lin, F., Marcu, D., Reiter, R., And Scherl, R. A Logical Approach to High-Level Robot Programming - A Progress Report. In Control of the Physical World by Intelligent Systems, Papers from the 1994 AAAI Fall Symposium, B. Kuipers, Ed. AAAI, New Orleans, November 1994, pp. 79-85.

[10] LeVesque, H. What is planning in the presence of sensing. In Proceedings of the National Conference on Artificial Intelligence (AAAI-96) (1996).

[11] LeVesque, H. J., Reiter, R., Lespérance, Y., Lin, F., AND SCHERl, R. B. GOlOG: A Logic Programming Language for Dynamic Domains. The Journal of Logic Programming (1996). To appear.

[12] Lifschitz, V. Computing Circumscription. In Proceedings of the Ninth International Joint Conference on Artificial Intelligence (IJCAI-85) (1985), pp. 121-127.

[13] Lin, F., AND Reiter, R. State Constraints Revisited. Journal of Logic and Computation 4, 5 (1994). Special Issue on Actions and Processes.

[14] Lin, F., AND Shoham, Y. Concurrent Actions in the Situation Calculus. In Working Notes of the 4th International Workshop on Nonmonotonic Reasoning (1992), pp. 133-138.

[15] Manna, Z., And Pnueli, A. The Temporal Logic of Reactive and Concurrent Systems. Springer-Verlag, New York, 1992.

[16] McCarthy, J. Programs with Common Sense. In Semantic Information Processing, M. Minsky, Ed. The MIT Press, 1968, ch. 7, pp. 403-418.

[17] MCCarthy, J. Applications of Circumscription to Formalizing Commonsense Knowledge. Artificial Intelligence 28 (1986), 89-116. 
[18] McCarthy, J., And Hayes, P. J. Some Philosophical Problems from the Standpoint of Artificial Intelligence. In Machine Intelligence 4, B. Meltzer and D. Michie, Eds. Edinburgh University Press, Edinburgh, Scotland, 1969, pp. 463-502.

[19] McDermott, D. A Temporal Logic for Reasoning About Processes and Plans. Cognitive Science 6 (1982), 101-155.

[20] Miller, R. A Case Study in Reasoning about Actions and Continuous Change. In Proceedings ECAI (1996), John Wiley \& Sons.

[21] Miller, R. Notes on deductive and abductive planning in the event calculus. http//wwwlp.doc.ic.ac.uk/UserPages/staff/rsm/abstract14.html, July 1996.

[22] Miller, R., And Shanahan, M. Narratives in the Situation Calculus. The Journal of Logic and Computation 4, 5 (1994), 513-530.

[23] Pinto, J. Concurrent Events: Synergy and Cancellation of Effects. In European Conference on Artificial Intelligence, Workshop on Logic and Change (1994), pp. 105-110.

[24] Pinto, J. Temporal Reasoning in the Situation Calculus. PhD thesis, Department of Computer Science, University of Toronto, Toronto, Ontario, Canada, Feb. 1994. URL = ftp://ftp.cs.toronto.edu//cogrob/jpThesis.ps.Z.

[25] Pinto, J. Integrating discrete and continuous change in a logical framework. Submitted for Publication, 1996.

[26] PinTo, J. State constraints and epistemological completeness. In progress, 1996.

[27] Pinto, J., And Reiter, R. Adding a Time Line to the Situation Calculus. In The Second Symposium on Logical Formalizations of Commonsense Reasoning (Jan. 1993), pp. 172177.

[28] Pinto, J., And Reiter, R. Temporal Reasoning in Logic Programming: A Case for the Situation Calculus. In Proceedings of the Tenth International Conference on Logic Programming (Budapest, June 1993), D. S. Warren, Ed., The MIT Press, pp. 203-221.

[29] Pinto, J., And Reiter, R. Reasoning about Time in the Situation Calculus. Annals of Mathematics and Artificial Intelligence 14, 2-4 (September 1995), 251-268.

[30] Pinto, J. A. On the Existence and Formalization of Natural Events. In Proceedings of the IJCAI Workshop on Nonmonotonic Reasoning, Action and Change. (Aug. 1995).

[31] ReIter, R. The Frame Problem in the Situation Calculus: A Simple Solution (sometimes) and a completeness result for goal regression. Artificial Intelligence and Mathematical Theory of Computation: Papers in Honor of John McCarthy. Academic Press, San Diego, CA, 1991, pp. 359-380.

[32] ReIter, R. Formalizing database evolution in the situation calculus. In Proceedings of the International Conference on Fifth Generation Computer Systems (June 1992), pp. 600-609.

[33] ReIter, R. Proving Properties of States in the Situation Calculus. Artificial Intelligence 64, 2 (Dec. 1993), 337-351. 
[34] Reiter, R. Natural Actions, Concurrency and Continuous Time in the Situation Calculus. In Proc. Common Sense 96: 3rd Symposium on Logical Formalizations of Commonsense Reasoning (Stanford, CA, Jan. 1996).

[35] Scherl, R., And Levesque, H. The Frame Problem and Knowledge Producing Actions. In Proceedings AAAI-93 (Washington, D.C., July 1993), AAAI, pp. 689-695.

[36] Shanahan, M. A Circumscriptive Calculus of Events. Artificial Intelligence 77, 2 (September 1995), 249-284.

[37] Shanahan, M. P. Solving the Frame Problem: A Mathematical Investigation of the Common Sense Law of Inertia. MIT Press, 1996. To appear.

[38] Widom, J., And Ceri, S. Active Database Systems, Triggers and Rules for Advanced Database Processing. Morgan Kaufmann Publishers, Inc., 1996.

[39] Zaniolo, C. Active database rules with transaction-conscious stable-model semantics. In Lecture Notes in Computer Science: Deductive and Object-Oriented Databases (Dec. 1995), T. W. Ling, A. Mendelzon, and L. Vieille, Eds., Springer Verlag. 